\title{
Liver macrophages contribute to pancreatic cancer-related cachexia
}

\author{
MARC E. MARTIGNONI ${ }^{1 *}$, CORNELIU DIMITRIU ${ }^{1 *}$, JEANINNE BACHMANN $^{1}$, HOLGER KRAKOWSKI-ROSEN $^{2}$, \\ KNUT KETTERER $^{1}$, RALF KINSCHERF ${ }^{3}$ and HELMUT FRIESS ${ }^{1}$ \\ ${ }^{1}$ Department of Surgery, Klinikum rechts der Isar, Technische Universität München, Munich; \\ ${ }^{2}$ German Cancer Research Center, Heidelberg; ${ }^{3}$ Department of Anatomy and Developmental \\ Biology, Medical Faculty Mannheim, University of Heidelberg, Germany
}

Received July 6, 2008; Accepted August 25, 2008

DOI: $10.3892 /$ or_00000231

\begin{abstract}
Cachexia is a devastating process especially in pancreatic cancer patients and contributes to their poor survival. We attempted to clarify the pathological and molecular changes that occur in the liver during the development of cachexia. Using immunohistochemistry we investigated the infiltration of inflammatory mononuclear cells in liver biopsies of pancreatic cancer patients with or without cachexia, and the potential relevance of the cells for the nutritional and inflammatory status. Additionally, these findings were compared with the patients' clinical parameters. We found a significantly higher amount of CD68 immunoreactive macrophages in liver cross sections of patients with pancreatic cancer and cachexia. The number of CD68-positive macrophages was significantly inversely correlated with the nutritional status. Additionally, in these CD68-positive areas a significant increase in IL-6 and IL-1 immunoreactive cells was localized. Moreover, we found significantly increased areas of CD68-positive macrophages in liver biopsies of patients with a more dedifferentiated (aggressive) grading of the tumor. In conclusion, these results suggest that a crucial interaction between the tumor, PBMCs, and the liver may play a central role in the development and regulation of cachexia. Furthermore, pancreatic cancer may be able to alter systemic organ function even without obvious metastatic disease.
\end{abstract}

\section{Introduction}

With a 5-year survival rate of $<5 \%$ and a death-to-incidence ratio of 0.99 , pancreatic ductal adenocarcinoma (PDAC) is

Correspondence to: Dr Marc E. Martignoni, Department of Surgery, Klinikum rechts der Isar, Technical University Munich, Ismaninger Str. 22, 81675 Munich, Germany

E-mail:martignoni@chir.med.tu-muenchen.de

${ }^{*}$ Contributed equally

Key words: pancreatic cancer, cachexia, liver, macrophages, grading currently one of the most aggressive gastrointestinal carcinomas (1-3). A variety of molecular alterations contributing to pancreatic cancer have been identified $(4,5)$. In spite of this, the reason for the aggressiveness of this disease remains unclear and the causes are multifactorial.

Furthermore, $>80 \%$ of PDAC patients exhibit cachexia, a complex metabolic and nutritional syndrome that is characterized by anorexia and a massive loss of adipose tissue and skeletal mass (6). Morbidity and mortality in pancreatic cancer are strongly influenced by the presence of cachexia, mainly due to severe respiratory muscle impairment and compromised immunity as a result of the massive loss of proteins (7). Therefore, cachectic PDAC patients have shorter survival and lower quality of life than patients with stable weight (8). Furthermore, cachexia already has an impact on the survival of patients with pancreatic cancer who are scheduled for tumor resection (9).

There is currently no generally accepted definition of cachexia. However, an unintentional loss of $>10 \%$ of total body weight within 6 months is generally accepted as a strong indicator for cachexia (10). Several theories have been suggested for the development of cachexia. One points to leptin, a hormone secreted by adipocytes. Leptin can reduce appetite and increase energy expenditure by interacting with several distinct hypothalamic neuropeptides, such as orexigenic NPY (neuropeptide Y) or anorexigenic CRF (corticotropin-releasing factor), melanocortin or neurotensin $(11,12)$. Thus, any abnormal process which induces or mimics the hypothalamic effect of leptin might produce anorexia and weight loss (13).

Other theories postulate the presence of tumor-derived compounds, such as PIF (proteolysis-inducing factor) and LMF (lipid-mobilizing factor), which may be responsible for the massive loss of muscular and adipose tissues, respectively $(14,15)$. Despite their differences, all these theories agree on two main concepts: on the one hand, that cachexia is characterized by a prolonged acute phase response in which liver protein synthesis changes from production of normal export proteins such as albumin to production of compounds such as C-reactive protein (CRP), $\alpha 1$-antitrypsin and fibrinogen; and on the other hand, that the de-novo production of cytokines such as TNF $\alpha$, IL-1ß, IL-6 
Table I. Demographic characteristics of PDAC patients with or without cachexia.

\begin{tabular}{lccr}
\hline & Cachexia $(\mathrm{n}=13)$ & No cachexia $(\mathrm{n}=14)$ & P-value \\
\hline Gender (n) & & & 0.685 \\
Male & $8(61.5 \%)$ & $10(71.4 \%)$ & \\
Female & $5(38.5 \%)$ & $4(28.6 \%)$ & 0.185 \\
Age (a) & $68(58 / 77)$ & $63(50 / 69)$ & 0.458 \\
ASA (n) & & & \\
II & $4(30.8 \%)$ & $8(57.1 \%)$ & 0.905 \\
III & $7(53.8 \%)$ & $6(42.9 \%)$ & \\
IV & $1(7.7 \%)$ & & $<0.001$ \\
Diabetes preoperative & $8(61.5 \%)$ & $9(64.3 \%)$ & $<0.001$ \\
No & $5(38.5 \%)$ & $5(35.7 \%)$ & 0.029 \\
Yes & $11.0(8.0 / 15.0)$ & $2.0(0 / 4.3)$ & \\
Weight loss $(\mathrm{kg})$ & $14.6(10.0 / 19.9)$ & $24.39(24.09 / 27.69)$ & \\
Weight loss $(\%)$ & $23.12(22.02 / 25.67)$ & & \\
BMI & & & \\
\end{tabular}

and IL- 8 are linked to the ongoing stimulus in the development of cachexia $(14,16)$.

Previous investigations identified some important new factors in the development of cachexia in pancreatic cancer. It was shown that tumor cells and peripheral blood mononuclear cells (PBMCs) produce high quantities of proinflammatory interleukins in pancreatic cancer cachexia $(17,18)$. Additionally, in vitro PBMCs of pancreatic cancer patients have been shown to be sensitized and stimulated by IL-6-positive pancreatic cancer cells to produce IL-6 in large amounts (17). Furthermore, in vitro PBMCs of cancer patients induce an acute phase response in primary liver cell lines via an IL-6-dependent mechanism (18).

In light of these findings, the aim of this study was to further assess possible interactions between PBMCs/ histiocytes and the liver in pancreatic cancer patients with and without cachexia and to further investigate the possible central role of the liver in cachexia induction and regulation.

\section{Patients and methods}

Tissue collection from patients. Normal liver tissue samples were obtained by open liver biopsy from 30 patients, 15 with and 15 without cachexia, who underwent surgery for PDAC. Since there is no generally accepted definition, cachexia in pancreatic cancer patients was defined as unintended loss of $>10 \%$ of body weight in the 6 months before diagnosis. Additionally, liver samples were obtained from 10 patients with chronic pancreatitis who underwent pancreatic head resection and from 5 patients with resection of colon cancer. For use as controls, liver biopsies were obtained through an organ donor program from 5 previously healthy individuals who were free of any pancreatic or neoplastic disease.

Two of the 15 patients with PDAC and cachexia were excluded after the final histological diagnoses proved to be chronic pancreatitis and mucinous pancreatic carcinoma,

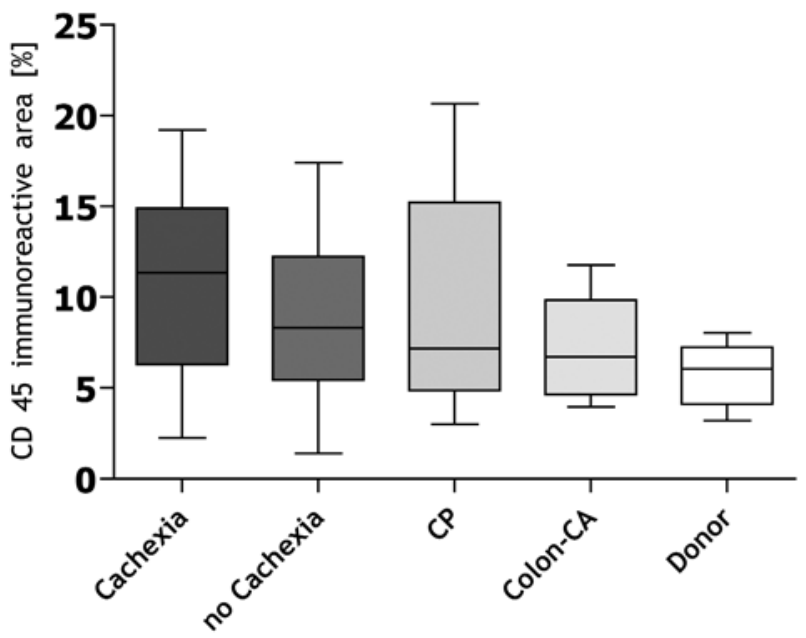

Figure 1. Box plots of percentage of CD45-immunoreactive cross sections of liver biopsies from patients with pancreatic adenocarcinoma with $(n=13)$ and without $(n=14)$ cachexia. Patients with chronic pancreatitis $(C P)(n=10)$, patients with colon cancer $(n=5)$ and normal donors $(n=5)$ served as controls.

respectively. One patient in the group with PDAC without cachexia was determined to have a papillary carcinoma and was also excluded.

The study was approved by the local ethics committee of the University of Heidelberg, Germany. All patients gave preoperative written informed consent for the use of their samples.

Immunohistochemistry. To immunohistochemically identify leukocytes or macrophages we used antibodies directed against CD68, a transmembrane protein expressed in monocytes and macrophages, and against CD45, a glycoprotein found in virtually all hematopoietic cells. All 


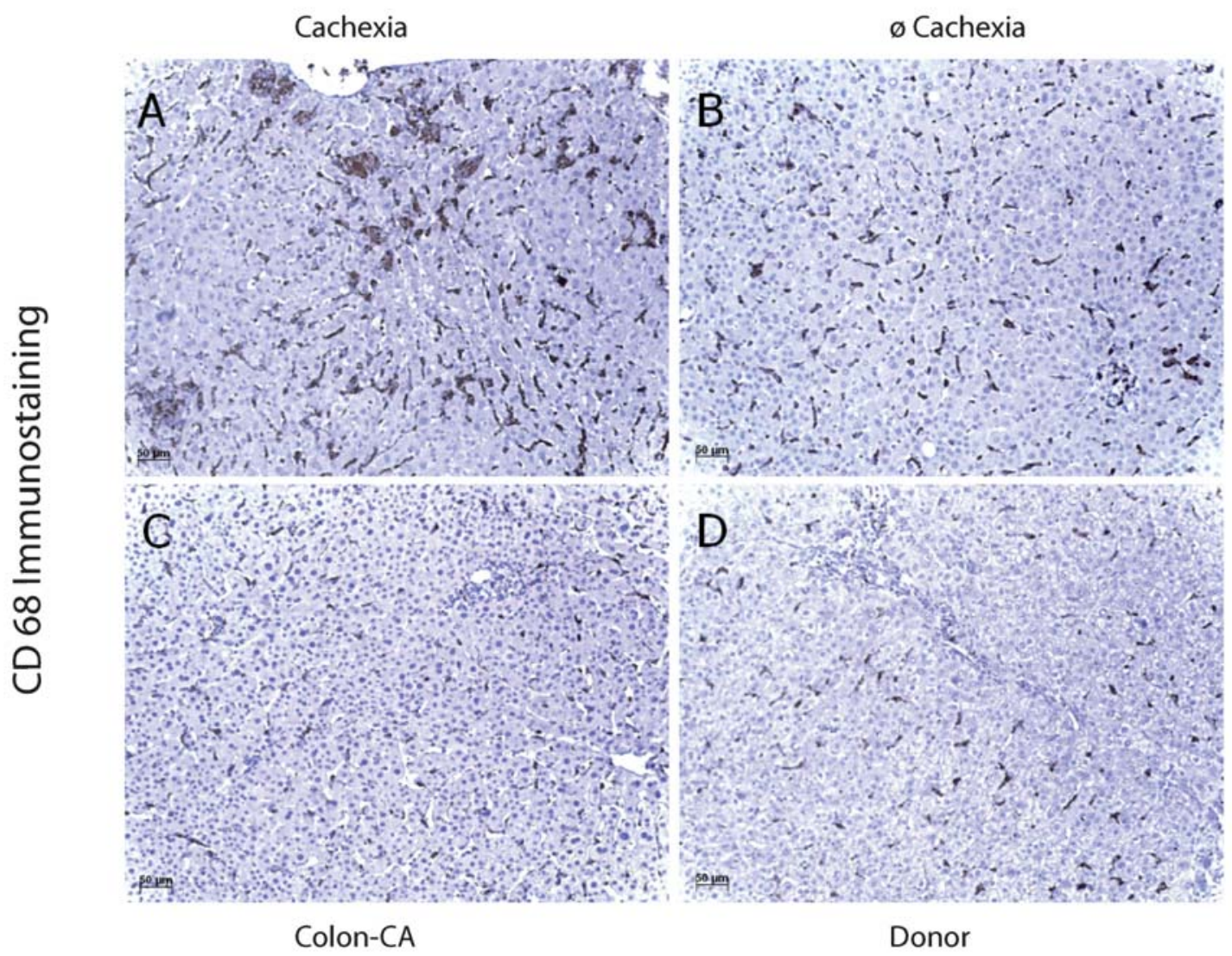

Figure 2. Immunohistochemistry of CD68-positive areas in cross sections of liver biopsies from a pancreatic cancer patient with cachexia (A) without cachexia; (B) a colon cancer patient; (C) and a normal donor and (D) with hematoxylin counterstaining. Magnification, x200.

freshly removed liver biopsies were immediately fixed in $5 \%$ paraformaldehyde solution for $24 \mathrm{~h}$, then embedded in paraffin, after which $3-\mu \mathrm{m}$ cross sections were processed for immunohistochemistry. Briefly, sections were deparaffinized in successive dilutions of ethanol. Heat-induced epitope retrieval was performed in a microwave oven using $10 \mathrm{mM}$ citrate buffer at $\mathrm{pH} 6.0$ for $20 \mathrm{~min}$. Endogenous peroxidase activity was blocked with $3 \%$ hydrogen peroxide. To block unspecific activity, tissues were treated with preimmune goat serum. Tissues were incubated with ready-to-use antibodies directed against CD68 or CD45. The sections were then incubated at $4^{\circ} \mathrm{C}$ overnight with mouse monoclonal primary antibody against CD68 or CD45 (both from Dako, Hamburg, Germany), IL-1 or IL-6 (both from Abcam Inc., Cambridge, MA, USA). After washing, an antimouse secondary antibody was applied for $45 \mathrm{~min}$. Dako Envision+ detection system was applied for signal visualization. Nuclei were counterstained with hematoxylin. In each experiment, incubation of cross sections without using the primary antibody was performed as a negative control.

Quantification of the stained cells. To allow quantification of the whole slide, the complete area of the slide was counted using the KS300 v. 3.0 software from Carl Zeiss Vision GMBH, Jena, Germany. Stained cells were counted as follows: all sections were scanned at x200 magnification and the total area and the stained area of each section were measured. Then the total stained area of the slide was measured by semi-automatic counting. The area of immunoreactive cells was expressed as the percentage of stained area out of the total area of the section.

Statistical analysis. Data were calculated as median, upper and lower quartile (median; 25th/75th) unless indicated otherwise. For statistical analysis, the Mann-Whitney test and the correlation according to Pearson and Spearman were performed using the statistical software package SPSS version 15.1 for Windows from SPSS Inc. (Chicago, IL, USA). A p $<0.05$ was considered as statistically significant.

\section{Results}

The preoperative characteristics of the PDAC patients with and without cachexia are summarized in Table I. There were no differences between the two groups regarding gender, age, ASA classification, or incidence of preoperative diabetes. Patients with PDAC and cachexia who had substantial weight loss and low BMI however reported feeling worse.

CD45 and CD68 immunohistochemistry of cross sections of liver biopsies. Although there was a tendency toward a higher infiltration rate of CD45-positive leukocytes in cross sections of liver biopsies of PDAC patients with cachexia, CD45-positive areas were similar in cross sections of liver biopsies of PDAC patients with $(11.34 ; 6.30 / 14.91)$ and without cachexia $(8.30 ; 5.45 / 12.23)$, patients with chronic 
Table II. Serum albumin, CRP, Hb and bilirubin levels as well as percentage of CD45, CD68 or IL-6 immunoreactive area in cross sections of liver biopsies of PDAC patients with and without cachexia.

\begin{tabular}{lrrr}
\hline & Cachexia $(\mathrm{n}=13)$ & No cachexia $(\mathrm{n}=14)$ & P-value \\
\hline Immunostaining $(\%)$ & & & \\
$\quad$ CD45 & $11.34(6.3 / 14.91)$ & $8.3(5.45 / 12.23)$ & 0.263 \\
CD68 & $6.96(3.67 / 9.90)$ & $3.68(2.84 / 6.48)$ & 0.044 \\
IL-6 & $13.16(8.57 / 27.77)$ & $6.51(5.25 / 12.88)$ & 0.048 \\
Albumin (g/l) & $41.05(39.03 / 42.28)$ & $43.7(41.1 / 46.85)$ & 0.068 \\
CrP (mg/l) & $6.6(2.6 / 22.2)$ & $9.0(4.9 / 20.6)$ & 0.769 \\
Hb (mg/dl) & $12.6(10.6 / 13.3)$ & $13.3(12.3 / 14.9)$ & 0.093 \\
Bilirubin (mg/dl) & $1.0(0.35 / 2.58)$ & $0.7(0.4 / 14.85)$ & 0.689 \\
\hline
\end{tabular}

Table III. Tumor load and features of patients with PDAC with or without cachexia.

\begin{tabular}{|c|c|c|c|}
\hline & Cachexia $(n=13)$ & No cachexia $(n=14)$ & P-value \\
\hline Tumor size (n) & & & 0.817 \\
\hline Stage 3 & $6(85.7 \%)$ & $12(92.3 \%)$ & \\
\hline Stage 4 & $1(14.3 \%)$ & $1(7.7 \%)$ & \\
\hline Lymph node (n) & & & 0.820 \\
\hline Negative & & $1(8.3 \%)$ & \\
\hline Positive & $6(100 \%)$ & $11(91.7 \%)$ & \\
\hline Metastasis (n) & & & 0.038 \\
\hline No & $6(46.2 \%)$ & $13(92.9 \%)$ & \\
\hline Yes & $7(53.8 \%)$ & $1(7.1 \%)$ & \\
\hline Grading (n) & & & 0.368 \\
\hline Moderate & $2(33.3 \%)$ & $8(61.5 \%)$ & \\
\hline Dedifferentiated & $4(66.7 \%)$ & $5(38.5 \%)$ & \\
\hline CA 19-9 (U/ml) & $1271.0(40.7 / 3253.0)$ & $418.5(59.18 / 2259.0)$ & 0.809 \\
\hline
\end{tabular}

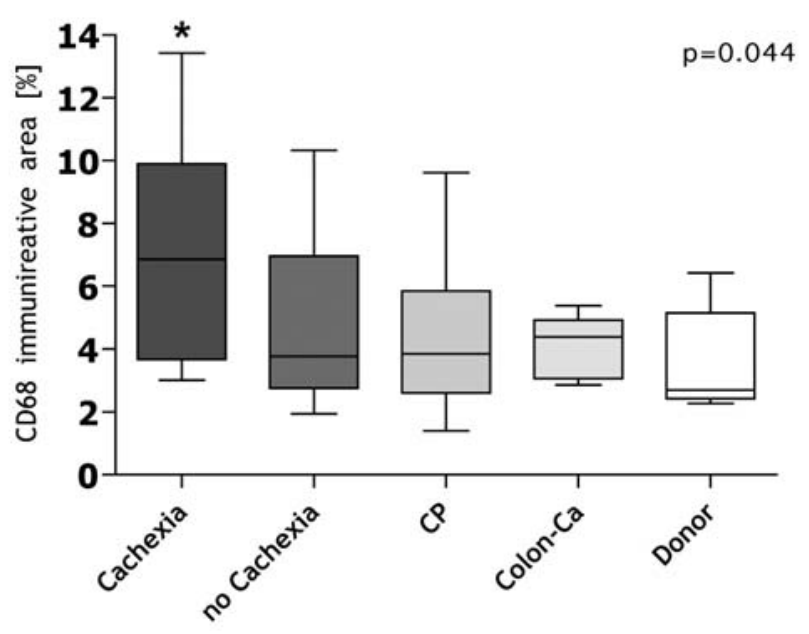

Figure 3. Box plots of percentage of CD68-immunoreactive cross sections of liver biopsies. The area of CD68-positive cells in normal liver tissue was significantly greater in patients with pancreatic cancer and cachexia than in patients without cachexia. Patients with chronic pancreatitis and colon cancer and normal donors served as controls. pancreatitis $(7.18 ; 4.86 / 15.22)$, colon cancer patients $(4.62$; $3.97 / 6.72)$ and normal donors $(4.11 ; 3.19 / 7.23)$ (Fig. 1; Table II). However, we found a significantly increased area of CD68positive macrophages in cross sections of liver biopsies from cachectic $(6.96 ; 3.67 / 9.90)$ compared to non-cachectic $(3.68$; 2.84/6.48) PDAC patients (Figs. 2 and 3; Table II). However, the percentage of CD68-positive areas in cross sections of liver biopsies from patients without cachexia was similar to that in liver cross sections from patients with chronic pancreatitis ( $3.84 ; 2.61 / 5.85)$, patients with colon cancer $(4.39 ; 3.06 /$ $4.935)$, or healthy donors $(2.69 ; 2.42 / 5.155)$, all of whom served as control groups (Fig. 3).

IL-6 and IL-1 $\beta$ immunohistochemistry in liver sections. In liver sections of cachectic PDAC patients, the percentage of IL-6-immunoreactive area was significantly $(p=0.048)$ increased $(13.16 ; 8.57 / 27.77)$ in comparison with noncachectic PDAC patients $(6.51 ; 5.25 / 12.88)$ or patients with chronic pancreatitis $(1.52 ; 0.64 / 9.52)$, colon cancer $(0.46$; $0.0 / 1.715)$ or donors $(0.04 ; 0.005 / 0.135)$ (Table II). However, 


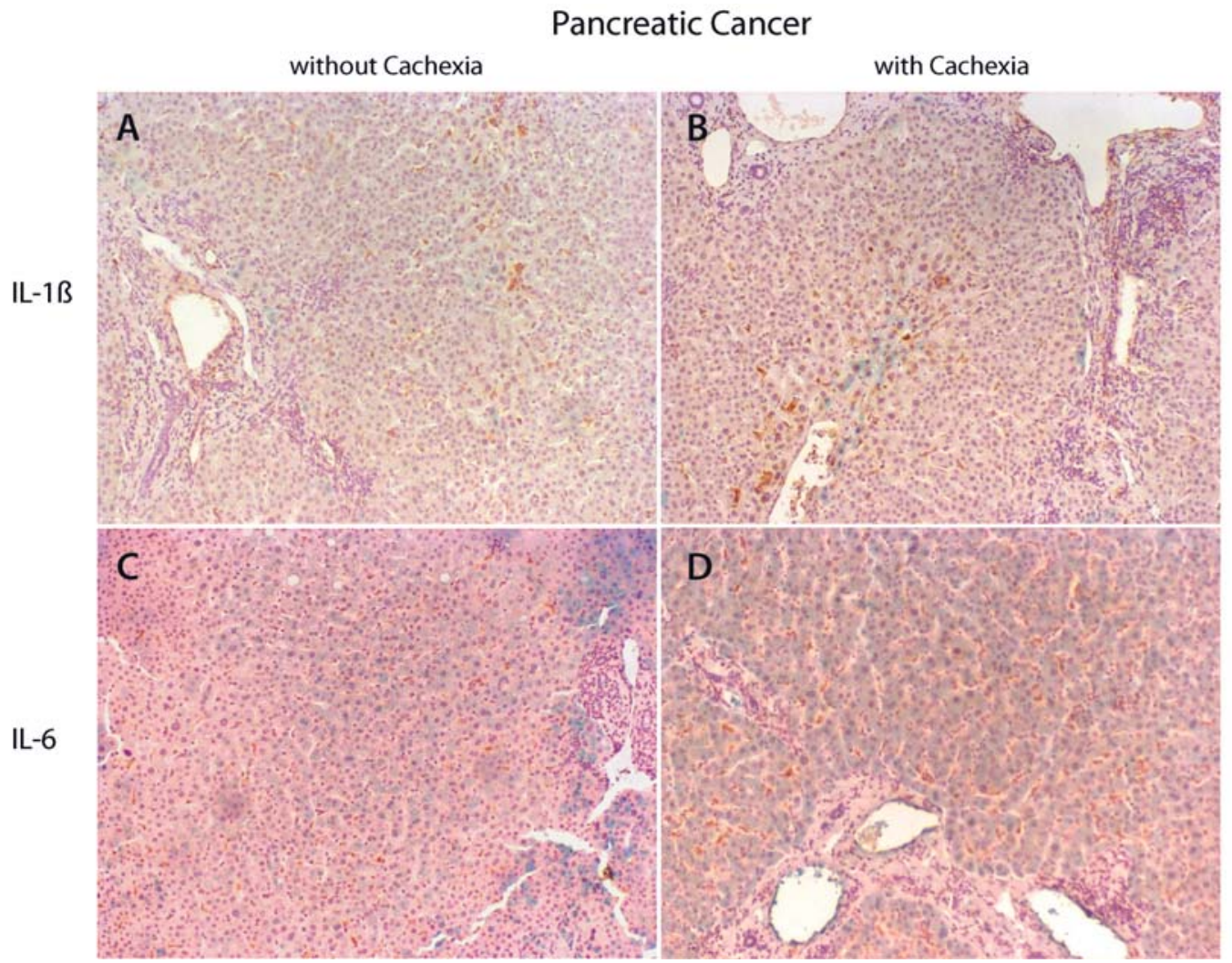

Figure 4. Immunohistochemistry in liver tissue of IL-1ß and IL-6 from patients with pancreatic adenocarcinoma without cachexia (A and D) and with cachexia (B and D). CD68 cells are stained in brown and IL-1B and IL- 6 are stained green. Magnification, x100.

A

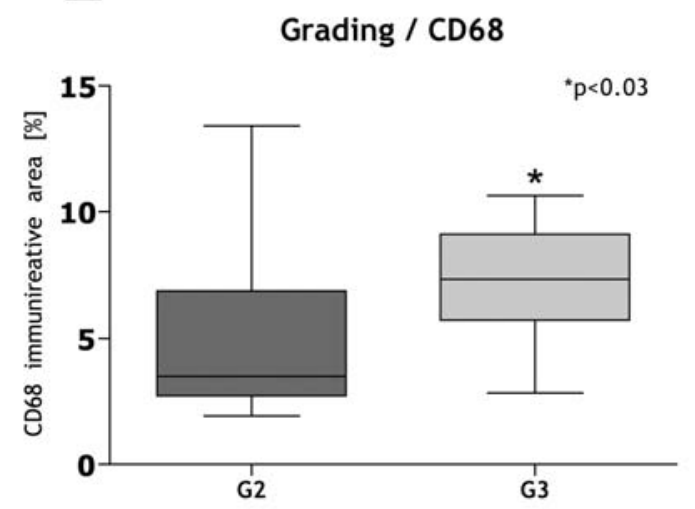

B

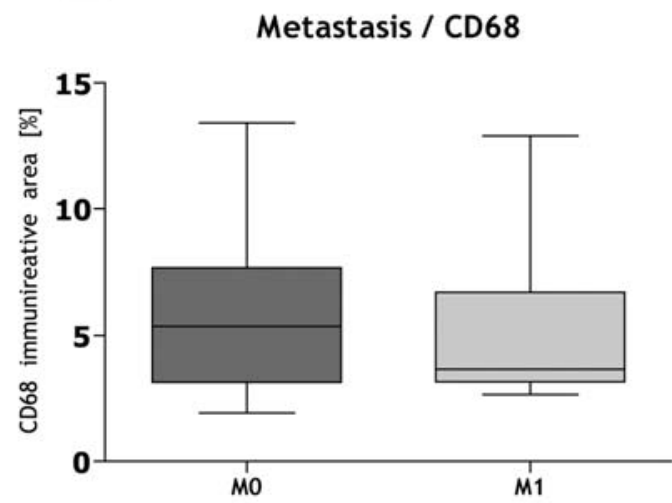

Figure 5. (A) Percentage of CD68-positive areas in PDAC patients are significantly higher for G3 tumors than for G2 tumors ( $\mathrm{n}=19$ ). (B) The presence of liver metastasis in patients with PDAC did not change the percentage of CD68-positive areas in liver biopsies of normal liver tissue.

IL-1ß immunostaining of liver sections was very faint in all groups, and thus could not be quantitatively evaluated. Double staining of liver sections revealed localization of CD68-positive macrophages close to IL-6-immunoreactive hepatocytes. CD68-positive macrophages and IL-6-immunoreactive hepatocytes were mainly localized in the portalvenous fields of the liver (Fig. 4).

Correlation with clinical parameters. PDAC patients with G3 tumors $(7.36 ; 5.76 / 9.14)$ exhibited a significantly increased percentage of CD68-immunoreactive areas in liver sections compared to patients with G2 tumors $(3.50 ; 2.73 / 6.89)(\mathrm{p}=0.03$; Fig. 5A). In contrast, patients with high and low tumor grades showed no difference in the percentage of CD45-immunoreactive area. However, there was no correlation between the percentage of CD68-immunoreactive area in liver sections and either tumor size, lymph node status or resectability of the tumor. In addition, no correlation with the presence of liver metastasis was found [CD68 in M0: $(5.37 ; 1.93 / 7.70)$ and in M1: $(3.67 ; 3.17 / 6.73)$ patients], although patients with cachexia had a higher incidence of metastatic disease (Table III; Fig. 5B). 
However, a significant positive correlation $(\mathrm{p}=0.04)$ between the percentage of CD68-positive area in liver cross sections and the serum $\mathrm{CrP}$ concentration was found for PDAC patients, although this significant correlation depends on one outlier (highest CrP level). Furthermore, the percentage of CD68-immunoreactive area in liver sections was significantly $(\mathrm{p}=0.019)$ inversely correlated with the total blood protein count and with albumin concentration in patients with and without cachexia.

In liver sections of PDAC patients, the percentage of IL-6immunoreactive area significantly correlated with the presence of cachexia $(\mathrm{p}=0.003)$ and the degree of weight loss $(\mathrm{p}=0.017)$, whereas no correlation was found with regard to nutritional status.

\section{Discussion}

Cachexia is a wasting condition with a significant impact on mortality and morbidity in pancreatic cancer (7). Despite intensive efforts to uncover its intricate pathways, the chain of events leading to cachexia is still not fully understood. Some of the main factors previously identified in the development of cachexia in pancreatic cancer are the tumor itself, PBMCs (peripheral blood mononuclear cells), as well as muscle and fat tissue $(14,17,19)$. Recent studies suggest that the liver may play an important role in the development of cancer cachexia, but the underlying mechanisms and the degree of involvement of the liver in the pathogenesis are still unclear $(18,20,21)$. Therefore, we investigated the histomorphologic changes in liver sections of PDAC patients with or without cachexia and correlated these findings with the clinical status of the patients. Up to now all available data concerning an interaction between the tumor and the liver are based on in vitro studies $(20,22)$. Therefore, our objective was to directly investigate the pathology of the normal liver in cancer patients and to view these results in the context of apparent changes in the liver tissue. As earlier and recent studies suggest an interaction of the liver and the tumor possibly mediated by mononuclear cells, we focused on infiltration of these cells into the liver in this context $(17,18,20)$. For this purpose we used the pan-leukocyte marker CD45 and the CD68 antibody to identify macrophages, such as those localized in the liver (liver macrophages, Kupffer cells) $(23,24)$. Whereas the leukocyte infiltration (identified by CD45 immunoreactivity) into the liver seemed not to be different in the groups tested, the number of CD68-positive macrophages was significantly higher in liver sections of cachectic PDAC patients in comparison with all other groups.

Based on these results, it is tempting to speculate that the number of infiltrating leukocytes and of macrophages is not different, but that in liver sections of cachectic PDAC patients the macrophages/Kupffer cells are specifically activated as indicated by CD68 immunoreactivity. This hypothesis may be confirmed by our finding of a positive relationship between the percentage of CD68-immunoreactive area in liver sections and the presence of cachexia in PDAC patients (Fig. 2A).

Recently it was shown that during hepatic immunoresponse to various insults, Kupffer cells are key initiators of inflammation (25). Therefore, according to our data, the presence of an increased number of CD68-positive macrophages in the liver suggests that this organ may play a much more important role in the development of cachexia in PDAC patients than has been suspected up to now. These findings are supported by in vitro experiments of Watchorn et $\mathrm{al}$, showing that tumor-derived factors such as the muscle proteolytic proteoglycan PIF are able to trigger the release of TNF- $\alpha$, IL- 6 and IL- 8 from isolated Kupffer cells. Subsequently, transcription factors such as nuclear factorkappa B $(\mathrm{NF}-\kappa \mathrm{B})$ and STAT3 are activated, which can further trigger neighboring hepatocytes to produce additional pro inflammatory cytokines and acute phase proteins (20). The amount of IL-6 staining in hepatocytes surrounding the CD68-positive liver macrophages was significantly greater in cachectic PDAC patients than in patients without cachexia, whereas IL-1ß staining in the liver was very faint in all groups, with a tendency to be more intense in cachectic patients. This suggests that in patients developing cachexia, liver parenchymal cells may be triggered by macrophages to produce pro-inflammatory cytokines like IL-6, whereas IL13 may play a more important role in muscle and fat tissue (Fig. 4).

In previous studies clinical parameters for liver function were assessed $(18,26)$. A strong inverse correlation with serum levels of albumin and total protein concentration was found, indicating a profound alteration of protein synthesis in the liver. With regard to these data, it provides further evidence that the liver might be one of the key factors in the development of cancer cachexia. This could be a further argument for the consideration of serum level of $\mathrm{CrP}$ as a comarker of cachexia in pancreatic cancer, as has been proposed in recently published clinical studies (26).

To check for specificity of this CD68 pattern in pancreatic cancer, normal liver tissue samples from patients with chronic pancreatitis and colon cancer were subsequently analyzed. All CD68-positive areas in liver tissues of pancreatic cancer patients without cachexia and in chronic pancreatitis and colon cancer patients were comparable with those found in the livers of normal donors, suggesting that pancreatic ductal adenocarcinoma and colon cancer do not entirely share the same pathways of cachexia (Fig. 2). However, the extent to which this CD68 liver pattern can be found in other digestive neoplasias is still a matter for further investigation.

To assess these liver features in the context of pancreatic cancer, the anatomical parameters of the tumor were analyzed. Patients with cachexia had a higher incidence of liver metastasis than patients without cachexia, and there was a tendency toward more dedifferentiated tumor grading (66.7 vs. $38.5 \%$ ), but tumor size, lymph node involvement and tumor load indicated by CA 19-9 were not significantly different (Table III). This may suggest that the development of cachexia is not necessarily related to the growth or size of the tumor, but is dependent on the aggressiveness of the tumor, as represented by histopathological grading. This is underlined by the finding that there was no relationship between percentage of CD68-positive areas and resection status, tumor size, lymph node involvement, or preoperative identification of liver metastases, but cachectic pancreatic 
cancer patients with G3 tumors exhibited significantly more enhanced CD68-positive areas in liver than patients with G2 tumors. Higher amounts of CD68-positive cells in liver correlated with the presence of cachexia, a finding that underlines the importance of histopathological grading of pancreatic cancer patients (8).

One of the most important prognostic parameters in pancreatic cancer is histopathological grading of the tumor. Mayer et al observed a significant correlation between tumor grading and survival time in a series of 113 patients, with 5-year survival rates of $33 \%$ for patients with G1 tumors, $10 \%$ for those with G2 tumors and $0 \%$ for those with G3 tumors. Furthermore, tumor grading appeared to be an independent factor in multivariate analysis (27). In view of these data, we may hypothesize that histopathological features of the tumor produce structural changes in the liver, which are subsequently related to cachexia. Since there was no correlation between these changes and the resection status, tumor size, lymph node involvement or preoperative identification of liver metastases, we can further hypothesize that, once in place, these structural modifications of the liver might be able to trigger and maintain a cachectic status, independent of the further presence of the tumor in the organism. Under these circumstances, we suggest pancreatic cancer to be a systemic disease, since it is able to produce structural alterations in organs other than the pancreas itself, independent of the presence of metastases, tumor size or lymph node involvement.

\section{Acknowledgements}

We wish to thank Mrs. Silke Vorwald for excellent technical assistance with immunohistochemistry and Mrs. Magdalena Geiss for excellent support during the study. This study was supported by a grant of the National Cancer Center Heidelberg-Mannheim.

\section{References}

1. Devesa SS, Blot WJ, Stone BJ, Miller BA, Tarone RE and Fraumeni JF Jr: Recent cancer trends in the United States. J Natl Cancer Inst 87: 175-182, 1995.

2. Friess H, Kleeff J, Kulli C, Wagner M, Sawhney $H$ and Buchler MW: The impact of different types of surgery in pancreatic cancer. Eur J Surg Oncol 25: 124-131, 1999.

3. Greenlee RT, Hill-Harmon MB, Murray T and Thun M: Cancer statistics, 2001. CA: Cancer J Clin 51: 15-36, 2001

4. Friess H, Berberat P, Schilling M, Kunz J, Korc M and Buchler MW: Pancreatic cancer: the potential clinical relevance of alterations in growth factors and their receptors. J Mol Med 74: 35-42, 1996.

5. Friess H, Guo XZ, Nan BC, Kleeff O and Buchler MW. Growth factors and cytokines in pancreatic carcinogenesis. Ann NY Acad Sci 880: 110-121, 1999.

6. Dewys WD, Begg C, Lavin PT, Band PR, Bennett JM, Bertino JR, Cohen MH, Douglass HO Jr, Engstrom PF, Ezdinli EZ, et al: Prognostic effect of weight loss prior to chemotherapy in cancer patients. Eastern Cooperative Oncology Group. Am J Med 69: 491-497, 1980.
7. Tisdale MJ: Molecular pathways leading to cancer cachexia. Physiology 20: 340-348, 2005.

8. Davidson W, Ash S, Capra S and Bauer J: Weight stabilisation is associated with improved survival duration and quality of life in unresectable pancreatic cancer. Clin Nutr 23: 239-247, 2004.

9. Bachmann J, Heiligensetzer M, Krakowski-Roosen H, Buchler MW, Friess H and Martignoni ME: Cachexia Worsens Prognosis in patients with resectable pancreatic cancer. J Gastrointest Surg 12: 1193-1201, 2008.

10. Kotler DP: Cachexia. Ann Intern Med 133: 622-634, 2000.

11. Friedman JM and Halaas JL: Leptin and the regulation of body weight in mammals. Nature 395: 763-770, 1998.

12. Inui A: Feeding and body-weight regulation by hypothalamic neuropeptides-mediation of the actions of leptin. Trends Neurosci 22: 62-67, 1999.

13. Inui A: Cancer anorexia-cachexia syndrome: are neuropeptides the key? Cancer Res 59: 4493-4501, 1999.

14. Tisdale MJ: Cachexia in cancer patients. Nat Rev 2: 862-871, 2002.

15. Tisdale MJ: Biomedicine. Protein loss in cancer cachexia. Science 289: 2293-2294, 2000.

16. McMillan DC, Scott HR, Watson WS, Preston T, Milroy R and McArdle CS: Longitudinal study of body cell mass depletion and the inflammatory response in cancer patients. Nutr Cancer 31: 101-105, 1998 .

17. Martignoni ME, Kunze P, Hildebrandt W, Kunzli B, Berberat P, Giese T, Kloters O, Hammer J, Buchler MW, Giese NA, et al: Role of mononuclear cells and inflammatory cytokines in pancreatic cancer-related cachexia. Clin Cancer Res 11: 5802-5808, 2005.

18. O'Riordain MG, Falconer JS, Maingay J, Fearon KC and Ross JA: Peripheral blood cells from weight-losing cancer patients control the hepatic acute phase response by a primarily interleukin-6 dependent mechanism. Int J Oncol 15: 823-827, 1999.

19. Inui A, Meguid MM: Cachexia and obesity: two sides of one coin? Curr Opin Clin Nutr Metab Care 6: 395-399, 2003.

20. Watchorn TM, Dowidar N, Dejong CH, Waddell ID, Garden OJ and Ross JA: The cachectic mediator proteolysis inducing factor activates NF-kappaB and STAT3 in human Kupffer cells and monocytes. Int J Oncol 27: 1105-1111, 2005.

21. Wigmore SJ, Fearon KC, Ross JA, McNally SJ, Welch WJ and Garden OJ: Febrile-range temperature but not heat shock augments the acute phase response to interleukin-6 in human hepatoma cells. Am J Physiol 290: G903-G911, 2006.

22. Watchorn TM, Waddell I, Dowidar N and Ross JA: Proteolysisinducing factor regulates hepatic gene expression via the transcription factors NF-(kappa)B and STAT3. FASEB J 15: 562-564, 2001.

23. Mytar B, Baran J, Gawlicka M, Ruggiero I and Zembala M: Immunophenotypic changes and induction of apoptosis of monocytes and tumour cells during their interactions in vitro. Anticancer Res 22: 2789-2796, 2002.

24. Lee WB, Erm SK, Kim KY and Becker RP: Emperipolesis of erythroblasts within Kupffer cells during hepatic hemopoiesis in human fetus. Anat Rec 256: 158-164, 1999.

25. Babu AN, Damle SS, Moore EE, Ao L, Song Y, Johnson JL, Weyant M, Banerjee A, Meng X and Fullerton DA: Hemoglobin-based oxygen carrier induces hepatic heme oxygenase 1 expression in Kupffer cells. Surgery 142: 289-294, 2007.

26. Fearon KC, Voss AC and Hustead DS: Definition of cancer cachexia: effect of weight loss, reduced food intake, and systemic inflammation on functional status and prognosis. Am J Clin Nutr 83: 1345-1350, 2006.

27. Meyer W, Jurowich C, Reichel M, Steinhauser B, Wunsch PH and Gebhardt C: Pathomorphological and histological prognostic factors in curatively resected ductal adenocarcinoma of the pancreas. Surg Today 30: 582-587, 2000. 